\title{
Comparisons of therapeutic effects of levodopa, levodopa and selegiline, and bromocriptine in patients with early, mild Parkinson's disease: three year interim report
}

\author{
Parkinson's Disease Research Group in the United Kingdom
}

\begin{abstract}
Objective-To determine the optimum treatment for early Parkinson's disease.

Design-An open, long term, prospective randomised trial conducted by the Parkinson's Disease Research Group of the United Kingdom.

Setting-93 hospitals throughout the United Kingdom.

Subjects-782 patients with early Parkinson's disease who were not receiving dopaminergic treatment.

Interventions-Patients allocated to treatment with levodopa/dopa decarboxylase inhibitor alone (arm 1), levodopa/decarboxylase inhibitor/selegiline in combination (arm 2), or bromocriptine (arm 3).

Main outcome measures-Disability assessment as judged by improvement on Hoehn and Yahr, modified Webster, and North Western University disability scales. Adverse event profile and mortality ratios.

Results-Interim results indicate that all three treatment regimens led to improvement in baseline disabilities after 12 months' treatment and that deterioration in control was apparent by three years. No significant differences were found between the results of treatment in arm 1 and arm 2 , but both were significantly more effective than bromocriptine (arm 3) and had fewer early adverse reactions. The adjusted difference $(95 \%$ confidence interval) in Webster rating for arm $3 v 1$ was 0.93 points $(0.27$ to $1.50 ; p=0.0058)$ and for arm $3 v 2$ was 1.25 points $(0.61$ to $1.89 ; p=0.0002)$. The incidence of dyskinesias and motor oscillations, however, was significantly lower in arm $3(2 \%$ and $5 \%$, respectively $)$ than in arm $1(27 \%$ and $33 \%$, respectively) and arm 2 (34\% and $35 \%$, respectively).
\end{abstract}

Conclusions-As there were no marked differences in functional improvement between the three groups the choice of treatment in the early stages of Parkinson's disease may not be critical.

\section{Introduction}

Although levodopa in combination with a dopa decarboxylase inhibitor is the single most effective treatment available for patients with Parkinson's disease, the long term therapeutic response is marred in many patients by the emergence of involuntary movements induced by the drug, oscillations in motor performance and mood, and psychotoxicity. ${ }^{1}$ As a result some physicians prefer to withhold levodopa, particularly in young patients, for as long as possible and elect to start treatment with an orally active dopamine receptor agonist, selegiline, an anticholinergic drug, or amantadine. ${ }^{2}$ Others advocate the early use of levodopa in all patients, claiming an improved life expectancy in comparison with those who have received the drug later in the course of their illness. ${ }^{3}$ The initiation of antiparkinsonian treatment with a $\vec{\omega}$ combination of an orally active dopamine receptor $\partial \vec{\partial}$ agonist and small doses of levodopa has also been 3 . recommended. ${ }^{4}$ Recently there has been interest in the possibility that controlled release preparations of levodopa may be less likely to induce long term \& complications than conventional preparations. ${ }^{5}$

Selegiline in a dose of $10 \mathrm{mg}$ a day can delay the need for levodopa in previously untreated patients; some of 6 this effect may result from a neuroprotective action 옥 on dopaminergic neurones. ${ }^{6}$ Concern has also been $N$ expressed on theoretical grounds that levodopa may increase formation of toxic free radicals in surviving dopaminergic neurones.

In an attempt to clarify some of these issues the Parkinson's Disease Research Group of the United $\ddot{\bullet}$ Kingdom, with a membership of consultant neurologists and a group of consultant physicians in geriatric medicine with a particular interest in Parkinson's disease, agreed to enter all newly diagnosed patients with idiopathic Parkinson's disease into an open, ्ֶ๊ long term three arm study comparing a conventional $\mathbb{D}$ preparation of levodopa/dopa decarboxylase inhibitor, $\overrightarrow{\vec{P}}$ levodopa/dopa decarboxylase inhibitor plus selegiline $\frac{\circ}{3}$ hydrochloride in combination, and bromocriptine alone. Our aim was to compare the therapeutic response, the profile of adverse reactions, and the mortality in the three groups of patients.

Fifty eight investigators entered patients from 93 hospitals. In the United Kingdom most patients with $\frac{5}{3}$ Parkinson's disease are referred to a hospital specialist for confirmation of the diagnosis, and we consider that the patients studied closely represent those seen in general practice. More than $90 \%$ of patients who were asked to participate in the study consented.

\section{Patients and methods}

Patients were eligible for the study if they fulfilled N the criteria of the Parkinson's Disease Society of the 0 United Kingdom Brain Tissue Bank for the clinical 0 diagnosis of Parkinson's disease. ${ }^{8}$ Untreated patients of any age with incapacity which in the judgment of $\stackrel{?}{?}$ the clinician concerned was sufficient to merit dopa- $T$ minergic treatment were eligible for inclusion. Patients who had previously received anticholinergic drugs $\stackrel{D}{\Omega}$ and those who had been considered on uncertain or $\mathbb{Q}$ incomplete evidence to be intolerant of levodopa were also considered suitable for entry. Those patients who were known with certainty to have failed to respond to dopaminergic drugs and those with incapacitating cognitive impairment, however, were excluded. The study received ethical approval from the Faculty of Clinical Sciences, University College and Middlesex Medical Schools. Some of the investigators also sought local ethical approval before starting the study. 
Expected mortality was based on the study by Shaw et al, who reported a $27 \%$ mortality after six years' follow up ${ }^{1}$ and $57 \%$ after 12 years' follow up ${ }^{9}$ in a group of British men and women treated with levodopa for Parkinson's disease.

It was calculated that about 600 people would need to be followed up for 10 years to detect a $30 \%$ reduction in mortality (power $80 \%$, significance $5 \%$ ). This figure was chosen because in an open uncontrolled retrospective survey Birkmayer et al had shown a 30\% reduction in mortality for a group of patients taking levodopa and selegiline compared with a group of patients taking levodopa alone. ${ }^{10}$

A total of 782 patients were recruited up to September 1990 to allow for possible loss to follow up. This sample size was more than adequate for detecting a one point difference on the modified 12 point Webster rating scale. ${ }^{11}$ The group considered it to be impractical and unethical to conduct a controlled double blind study over the projected length of follow up of many years.

The randomisation of the patients into one of three treatment regimens was carried out by a centrally based administrative coordinator by using random numbers tables. ${ }^{12}$ Treatment 1 consisted of $62.5 \mathrm{mg}$ of levodopa and benserazide three times a day after meals. The dose was then increased to $125 \mathrm{mg}$ thrice daily and maintained for at least three months before further increases were considered. Details of further increments and maintenance were then left to the investigator, but the aim of treatment was to achieve a satisfactory improvement in symptoms and functional disabilities by using the lowest possible dose. Treatment 2 was started with $5 \mathrm{mg}$ selegiline in the morning for a week followed by an increase to $5 \mathrm{mg}$ twice daily for three weeks. Clinical experience indicates that a twice daily regimen of selegiline is better tolerated than a single $10 \mathrm{mg}$ daily dose. Levodopa and benserazide were then added in the same way as for treatment 1 , selegiline being continued at the same dosage. In treatment 3 bromocriptine was given alone starting with $2.5 \mathrm{mg}$ after the evening meal and increasing by $2.5 \mathrm{mg}$ no faster than every third day to a dose of $30 \mathrm{mg}$ daily administered in three divided doses with meals. ${ }^{13}$ Increases no faster than $10 \mathrm{mg}$ weekly were then made up to a maximum dose of $40 \mathrm{mg}$ three times a day. If peripheral side effects occurred in any of the arms the investigator could prescribe domperidone, a peripheral dopamine antagonist, $20 \mathrm{mg}$ three times a day.

The principal outcome measures were mortality and assessment of disability. Patients were evaluated at baseline and then every three to four months, whenever possible by the consultant investigator and preferably in the presence of a close relative. Disability assessments were made by using the Hoehn and Yahr ${ }^{14}$ North Western University disability, ${ }^{15}$ and modified 12 item Webster rating scales $^{11}$ to include additional parameters for balance and rising from a chair; these rating scales were provided as printed copies. The occurrence of adverse reactions including involuntary movements, oscillations in performance, and early morning dystonia were also reported. During the titration period patients were seen every three to four weeks.

If a patient was unable to tolerate a trial drug or failed to gain useful overall improvement based on the clinician's judgment, he or she could either be re-randomised to one of the other two arms of the trial or withdrawn from the trial. Patients have been considered in this report only in relation to their original randomisation. Patients withdrawn from the trial are followed up annually. Analyses of disabilities, incidence of side effects, and mortality were performed once a year. An independent analysis of inter-rater reliability was carried out, and no notable differences of scoring between investigators were found.

Differences among the treatment regimens were tested at a more stringent level of significance to allow for this repeated testing. No formal stopping rules were applied for disability scores; instead average changes in disability and incidence of side effects were monitored by the investigators. Analyses of this interim report are based on follow up of patients up to December 1991. The trial and monitoring of mortality continues.

The main analyses were conducted on an intention to treat basis in which individual subjects were allocated to their initial treatment group irrespective of whether they were subsequently withdrawn from randomised treatment or not. This avoids bias arising from selection of patients who continue to be treated and more closely reflects the clinical management, in which patients' treatment may be modified in the light of their response to initial treatment. It may, however, underestimate the magnitude of effect associated with a particular treatment. Inspection of average disability scores for all treatments combined showed an initial improvement in the first three months followed by a levelling off in the next four to 12 months, and after one year of follow up average scores displayed an approximately constant rate of decline. It was therefore decided to analyse disability scores by taking the average of all assessments in months four to 12 for each subject and then the average of the two most recent assessments for each subject (final score). The average of two or more assessments was used to improve the reliability of the disability measure.

As predicted in the design of the study, ${ }^{13}$ bromocriptine was not well tolerated by all patients, and a substantial number were withdrawn from the regimen in the first few months of follow up because of adverse side effects. Because it has been suggested that those patients who are able to tolerate bromocriptine may do better in the long term than patients initially treated with levodopa, an on treatment analysis was performed to examine this hypothesis. Bias relating to comparison of a subgroup of patients with more benign or milder disease, however, may be introduced with this method of analysis.

End points were also analysed to look for possible associations with recorded entry characteristics such as age, sex, reported duration of Parkinson's disease, and the Hoehn and Yahr stage. The possibility that differences in treatment could be related to these factors was also examined. Analysis of covariance was used to assess the difference in disability scores between the three treatments. This method takes account of any differences in average baseline scores present in the three treatment groups. In the analysis of final score months of follow up was also included in the model to adjust for any difference in duration of follow up among the three groups of treatment.

\section{Results}

Table I shows that the three groups were comparable in terms of recorded baseline characteristics. The mean (range) duration of disease before treatment was 14 (1-144) months with no significant difference between the three arms. Although the median doses of levodopa after one year's treatment did not differ between arms 1 (375 mg; range $125-1000 \mathrm{mg} /$ day) and 2 (375 $\mathrm{mg} ; 62.5-1000 \mathrm{mg} /$ day), there was a significant difference in the mean doses $(420 \mathrm{mg} /$ day in arm $1 v$ $352 \mathrm{mg} /$ day in arm $2 ; \mathrm{p}=0.05$ ), possibly indicating that the presence of selegiline reduces the need for levodopa. The mean dose of bromocriptine was $36 \mathrm{mg}$ a day with a wide range (7.5-120 mg/day).

A total of 43 patients had their diagnoses revised 


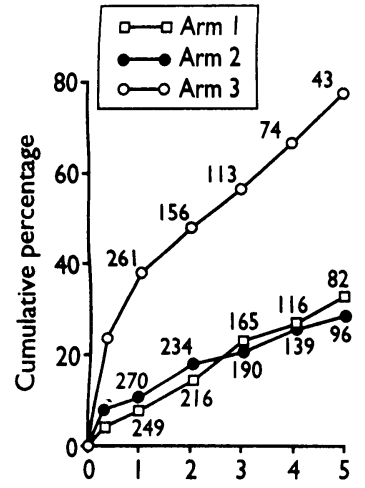

Years since entry to trial

FIG 1 -Cumulative percentages of patients with Parkinson's disease who were withdrawn from randomised treatment. The numbers are those at risk for each year since entry during the course of the trial. Patients with revised diagnoses are included in the analysis to reflect clinical practice, in which some patients will inevitably be treated for Parkinson's disease before having the correct disease diagnosed. The new diagnoses were multiple system atrophy (13 cases), severe dementia (Lewy body or Alzheimer's disease; 10), SteeleRichardson-Olszewski disease (six), essential tremor (two), masked depression (two), communicating hydrocephalus (one), frontal meningioma (one), writers' cramp (one), and not specified (seven). The common causes for withdrawal because of adverse reactions were gastrointestinal (four in arm 1, 14 in arm 2,38 in arm 3) and psychiatric (none in arm 1, three in arm 2,10 in arm 3). Early adverse reactions were fewest in arm 1. Table II shows the reasons for withdrawal in each arm. Protocol violators were defined as those patients whose regimen was changed by a physician other than a trial investigator. The most common violation was the introduction of selegiline to arm 1 after the initial favourable report of the DATATOP study. ${ }^{6}$ Figure 1 indicates the cumulative percentage withdrawal for the three arms.

Analysis indicated that of the three scales used the modified Webster score was the most sensitive measure of disability, and the results in this paper are based on this scale. The trend was similar with both of the other scales. The average improvement in Webster disability score during the first year of follow up was significantly greater in arms 1 and 2 compared with arm 3 (table III). The differences between arm 1 and arm 3 and between arm 2 and arm 3 were significant (table IV). There was no significant difference between arms 1 and $2(p=0.35)$.

Figure 2 shows the change in score by time since entry to trial. The average rate of change in the Webster score after the first year of follow up was 0.7 points a year. Differences in final score among treatment regimens were similar to those reported

TABLE I-Baseline characteristics of patients with Parkinson's disease entered into trial of different treatments

\begin{tabular}{|c|c|c|c|}
\hline & Arm 1 & Arm 2 & Arm 3 \\
\hline No entered $\star$ & 249 & 271 & 262 \\
\hline No of men & 138 & 145 & 148 \\
\hline No of women & 111 & 126 & 114 \\
\hline Age (years): & & & \\
\hline $\begin{array}{l}\text { Mean } \\
\text { Overall SD (range) }\end{array}$ & $62 \cdot 7$ & $\begin{array}{c}63 \cdot 6 \\
9 \cdot 8(25-86)\end{array}$ & $62 \cdot 1$ \\
\hline North Western University disability score: & & & \\
\hline $\begin{array}{l}\text { Mean } \\
\text { Overall SD (range) }\end{array}$ & 43.9 & $\begin{array}{c}42 \cdot 8 \\
5 \cdot 3(50-19)\end{array}$ & $42 \cdot 9$ \\
\hline Webster rating: & & & \\
\hline $\begin{array}{l}\text { Mean } \\
\text { Overall SD (range) }\end{array}$ & $11 \cdot 0$ & $\begin{array}{r}11 \cdot 8 \\
4 \cdot 7(1-29)\end{array}$ & 11.9 \\
\hline Hoehn and Yahr state (\%): & & & \\
\hline I & 27.5 & $22 \cdot 1$ & $25 \cdot 1$ \\
\hline II & $40 \cdot 0$ & $43 \cdot 2$ & $40 \cdot 2$ \\
\hline III & $29 \cdot 1$ & $29 \cdot 2$ & $29 \cdot 7$ \\
\hline IV and $\mathrm{V}$ & $3 \cdot 7$ & 5.5 & $5 \cdot 0$ \\
\hline
\end{tabular}

*Total of 782 subjects entered between May 1981 and September 1990 Arm 1: levodopa/dopa decarboxylase inhibitor.

Arm 2: levodopa/dopa decarboxylase inhibitor/selegiline. Arm 3: bromocriptine.

TABLE II-Reasons for withdrawal from trial of different treatment for Parkinson's disease as proportion of withdrawals from each treatment. Figures are numbers of patients

\begin{tabular}{lccc}
\hline & $\begin{array}{c}\text { Arm 1 } \\
\text { (249 patients } \\
\text { entered, } \\
\text { Reason for } \\
\text { withdrawal }\end{array}$ & $\begin{array}{c}\text { Arm 2 } \\
\text { (271 patients } \\
\text { entered, }\end{array}$ & $\begin{array}{c}\text { Arm 3 } \\
\text { (263 patients } \\
\text { entered, }\end{array}$ \\
\hline Lost to follow up & 15 & 12 & 3 \\
Poor compliance & 8 & 14 & 7 \\
Protocol violation & 22 & 2 & 41 \\
Lack of response & 2 & 1 & 30 \\
Deterioration & 11 & 12 & 69 \\
Adverse reaction & 6 & 22 & 22 \\
Revised diagnosis & 16 & 13 & \\
\hline
\end{tabular}

Arm 1: levodopa/dopa decarboxylase inhibitor.

Arm 2: levodopa/dopa decarboxylase inhibitor/selegiline.

Arm 3: bromocriptine.
TABLE III-Improvement in Webster rating during first year of follow up in patients with Parkinson's disease according to different treatments

\begin{tabular}{lccc}
\hline & Arm 1 & Arm 2 & Arm 3 \\
\hline No included in analysis & 213 & 233 & 224 \\
Pooled baseline score & & $11 \cdot 7$ & \\
Adjusted improvement in score & $3 \cdot 1$ & $3 \cdot 4$ & $2 \cdot 1$ \\
\hline
\end{tabular}

Arm 1: levodopa-dopa carboxylase inhibitor.

Arm 2: levodopa/dopa carboxylase inhibitor/selegiline.

Arm 3: bromocriptine.

TABLE $\mathrm{IV}-$ Adjusted differences in Webster rating between different treatments during first year of follow up in patients with Parkinson's disease

\begin{tabular}{lcl}
\hline Comparison & $\begin{array}{c}\text { Adjusted difference } \\
(95 \% \text { confidence interval) }\end{array}$ & p Value \\
\hline Arm $1 v 2$ & $0.31(-0.34$ to 0.97$)$ & 0.35 \\
Arm $3 v 1$ & $0.93(0.27$ to 1.50$)$ & 0.0058 \\
Arm $3 v 2$ & $1.25(0.61$ to 1.89$)$ & 0.0002 \\
\hline
\end{tabular}

${ }^{\star}$ Positive difference indicates disadvantage for first treatment compared with second.

Arm 1: levodopa/dopa carboxylase inhibitor.

Arm 2: levodopa/dopa carboxylase inhibitor/selegiline.

Arm 3: bromocriptine.

TABLE V-Numbers (percentages) of patients in each group with side effects *

\begin{tabular}{lccc}
\hline Side effects & $\begin{array}{c}\text { Arm 1 } \\
(\mathrm{n}=249)\end{array}$ & $\begin{array}{c}\text { Arm 2 } \\
(\mathrm{n}=271)\end{array}$ & $\begin{array}{c}\text { Arm 3 } \\
(\mathrm{n}=263)\end{array}$ \\
\hline Dyskinesia & $67(27)$ & $92(34)$ & $5(2)$ \\
Dystonia & $63(25)$ & $65(24)$ & $18(7)$ \\
Oscillations & $82(33)$ & $95(35)$ & $13(5)$ \\
None & $137(55)$ & $133(49)$ & $234(89)$ \\
\hline
\end{tabular}

*Some patients had more than one side effect.

Arm 1: levodopa/dopa carboxylase inhibitor.

Arm 2: levodopa/dopa carboxylase inhibitor/selegiline.

Arm 3: bromocriptine.

during the first year of follow up. No evidence was found that the average rate of decline after the first year of follow up differed among the three treatment groups, and the interaction between baseline disability score and treatment group was not significant. The on treatment analysis indicates that subjects remaining in arm 3 for at least one year still show less improvement than those remaining in arms 1 and 2 although the magnitude of difference is slightly reduced. The mean length of time to develop dyskinesias and motor oscillations did not differ significantly between arms 1 and 2 (24.5 $v 29.0$ months). After an average follow up of three years the incidence of dyskinesias and disabling on-off oscillations induced by treatment was lower in arm $3(2 \%$ and $5 \%)$ than in arms $1(27 \%$ and $33 \%$ ) and $2(34 \%$ and $35 \%$ ) (table V). Age and baseline Hoehn and Yahr stages were significantly associated with both improvement in the first year $(p=0.0001$ and $p=0.47$, respectively) and final score. Tests for interactions between these two variables and the treatment group were not significant.

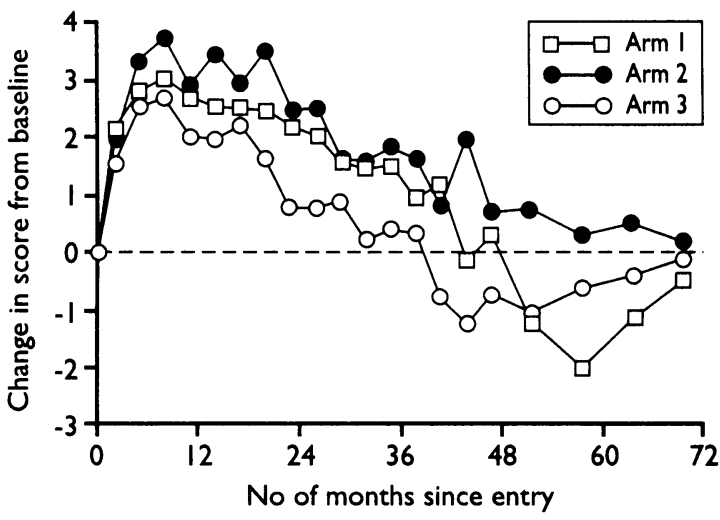

FIG 2-Average change in Webster score by treatment group in patient with Parkinson's disease 


\section{Discussion}

Analysis of this study after a mean of three years' follow up showed no significant difference between disability scores in patients receiving levodopa alone or in combination with selegiline. Significantly more adverse reactions, however, occurred in the levodopa plus selegiline group. The number of patients who have died is still small and longer follow up will be required to determine whether the addition of selegiline improves life expectancy. Patients receiving bromocriptine alone showed less improvement than those in the other two arms. Even the good responders who continued for at least one year did less well than the patients in the two groups treated with levodopa. The magnitude of difference, however, was small and equivalent to just one point on the modified Webster scale. In everyday functional terms this would probably indicate little disadvantage for bromocriptine alone over the first year or two of treatment. The number of early drop outs was significantly greater with bromocriptine, either because of an inadequate therapeutic response or because of intolerable side effects. On the other hand, those patients able to tolerate and benefit from bromocriptine experienced substantially less dyskinesia and oscillations in performance in the long term. No evidence to support a differential rate of decline among the treatment groups after initial improvement has been found. At this point in the trial most of the patients remain fairly mildly disabled and longer follow up is needed, even though the number of drop outs is likely to increase considerably as disability increases.

Most patients participating in the study started treatment within three years of the diagnosis having been established, and baseline disability scores were mild. The results suggest that whichever of the three treatment regimens is given, after three years of follow up average scores remain at or better than pretreatment level of disability. The choice of antiparkinsonian regimen, at least in the early stages of the disease, may not therefore be critical. Longer term follow up to assess disability and mortality may detect significant differences.

Members of the Parkinson's Disease Research Group of the United Kingdom are: $\mathrm{R}$ Abbott, $\mathrm{N}$ Banerii, M Barrie, $\mathrm{H}$ Boddie, P Bradbury, C Clarke, R Clifford-Jones, R Corston, E Critchley, P Critchley, R Cull, J Dick, I Draper, C Ellis, L Findley, T Fowler, A Gale, C Gardner-Thorpe, W Gibb, J D Gibson, J M Gibson, R Godwin-Austen, R Greenwood, R Hardie, D Harley, C Hawkes, S Hawkins, M HildickSmith, R Hughes, L Illis, J Jestico, K Kafetz, C Kennard, R Knight, R Kocen, A Lees, N Leigh, L Loizou, R Lenton, D MacMahon, C D Marsden, W Michael, J Mitchell, P Monro, J Moran, B Morgan, P Murdoch, W Mutch, P

\section{Clinical implications}

- Levodopa in combination with a peripheral dopa decarboxylase inhibitor is a more potent antiparkinsonian drug than bromocriptine for treating early mild Parkinson's disease and has fewer early adverse reactions

- The addition of selegiline to levodopa treatment produces no significant benefits over the first three years of treatment

- On/off oscillations and dyskinesias occur rarely with bromocriptine treatment alone

Overstall, D Park, J D Parkes, B Pentland, D Perkins, R Ponsford, N Quinn, M Rawson, J Rees, D Riddoch, F Schon, A Schapira, D Shepherd, G Stern, B Summers, C Turnbull, A Turner, $\mathrm{S}$ Vakil, C Ward, A Whiteley, A Williams.

We thank the Parkinson's Disease Society of the United Kingdom, Roche Products, Britannia Pharmaceuticals, and Sandoz Products for financial support, and Ms J Head, department of epidemiology and public health, University College and Middlesex School of Medicine, London, for statistical advice.

1 Shaw KM, Lees AJ, Stern GM. The impact of treatment with levodopa on Parkinson's disease. Of Med 1980;49:283-93.

2 Fahn S, Calne DB. Considerations in the management of Parkinsonism. Neurology 1978;28:5-7.

3 Markham CH, Diamond SG. Long-term follow-up of early dopa treatment in Parkinson's disease. Ann Neurol 1986;19:365-72.

4 Rinne UK. Early dopamine agonist therapy in Parkinson's disease. Mov Disord 1989;4(suppl 1):586-94.

5 Lees AJ. A hydrodynamically balanced controlled-release $L-$ dopa/benserazide formulation in the treatment of Parkinson's disease. In: Fahn S, Marsden CD, Calne DB, Goldstein M, eds. Recent developments in Parkinson's disease Vol 2. New Jersey: Macmillan Health Care Information, 1988.

6 Parkinson Study Group. Effect of deprenyl on the progression of disability in early Parkinson's disease. $N$ Engl f Med 1989;321:1364-71.

7 Cohen G. The pathobiology of Parkinson's disease: biochemical aspects of dopamine neuron senescence. 7 Neural Transm 1983;19:89-103.

8 Gibb WRG, Lees AJ. The relevance of the Lewy body to the pathogenesis of idiopathic Parkinson's disease. I Neurol Neurosurg Psychiatry 1988;51:
ing idiopathic

9 Curtis L, Lees AJ, Stern GM. Effect of L-dopa on the course of Parkinson's disease. Lancet 1984;ii:211-2.

10 Birkmayer W, Knoll J, Riederer P, Youdim MBH, Hars V, Marton Increased life expectancy resulting from addition of L-deprenyl to Madopar treatment in Parkinson's disease: a long term study. F Neural Transm 1985;64:113-27.

11 Webster DD. Clinical analysis of the disability in Parkinson's disease. Modern Treatment 1968;5:257-82.

12 Bradford-Hill A. Principles of medical statistics. London: Lancet, 1971.

13 Lees AJ, Stern GM. Sustained bromocriptine therapy in previously untreated patients with Parkinson's disease. $f$ Neurol Neurosurg Psychiatry 1981;44: patients $1020-3$.

14 Canter CJ, De la Torre R, Mier M. A method of evaluating disabilities in patients with Parkinson's disease. 7 Nerv Ment Dis 1961;133:143-7.

15 Hoehn MM, Yahr MD. Parkinsonism: onset, progression and mortality. Neurology 1967;17:427-42.

(Accepted 9 fune 1993)

\section{A TEACHER WHO CHANGED MY PRACTICE \\ Learning to think}

My first lesson in practical thinking occurred at school during a physics lesson on friction.

"Well boys," began our genial physics master, "Let's sort out this friction business and think about what causes it. Firstly, you start by telling me facts about friction and I'll explain them my way."

One by one we provided facts about friction and, much to our surprise, he explained every one of our facts in terms of little green gremlins. We were all taken aback at what we considered to be a most unscientific attitude, but he was in fact teaching us about thinking not physics.

We said, "It is more difficult to move one rough surface over another."

He replied, "That's because gremlins stand up on rough surfaces and their bodies get entangled."

We said, "It becomes easier if we oil the two rough surfaces."
He replied, "The oil drowns the gremlins and they lie down dead."

We said, "It gets easier if you sandpaper the surfaces to make them smooth."

He replied, "That's simply because you've cut the gremlins heads off."

"But Sir, we don't believe in gremlins, what about the molecules and things ...?"

This teacher had taught us two major scientific lessons. Firstly, you should avoid getting locked in your own mental library of possible solutions to a problem. Secondly, however fantastic the explanation, as long as it is consistent-that is, the gremlins must do the same thing each time-you shouldn't discard the solution out of hand. You should keep it and test it further until a better one which explains even more presents itself.-JOHN ASBURY is a senior lecturer in anaesthesia in Glasgow 